\title{
Técnicas de Projeto para Atendimento aos Requisitos de Cobertura de Estações de FM
}

\author{
Paulo E. R. Cardoso, Member, IEEE; Yuzo Iano, Senior Member, IEEE; Silvio R. M. \\ Carvalho, Member, IEEE; Hermes J. Loschi, Member, IEEE; Rafael F. Pinheiro e Thiago V. Silva
}

\begin{abstract}
Presents the assessment of 16 FM stations coverage held in the metropolitan region of São Paulo, pointing methodologies for prediction and measurement adopted by Anatel, analyzing the coverage gaps and showing, by simulations, the variations that the design features can causing to the coverage of a station, directly linked to the quality of service.

index - coverage, fitting, design, siganatel
\end{abstract}

Resumo-Apresenta a avaliação da cobertura em 16 estações de FM na região metropolitana de São Paulo, mostrando as metodologias de predição e de medição da cobertura adotadas pela Anatel, analisando as falhas de cobertura e mostrando, por meio de simulações, que alterações nas características de projeto podem alterar a cobertura, diretamente ligada à qualidade do serviço prestado.

Palavras Chave - cobertura, enquadramento, projeto, siganatel

\section{INTRODUÇ̃̃O}

$\mathrm{O}$ Serviços de Radiodifusão devem ser livre, direta e gratuitamente recebidos pelos usuários. $\mathrm{O}$ poder concedente leva em conta, dentre outros itens, a área de cobertura e a população atendida quando da outorga destes serviços, cabendo à Agência Nacional de Telecomunicações Anatel - a aprovação e fiscalização das características técnicas das estações, garantindo a prestação do serviço de acordo com os parâmetros determinados pela legislação. Neste cenário, as entidades prestadoras dos serviços estão obrigadas a manter as instalações de suas estações em condições de atendimento aos requisitos dos Regulamentos Técnicos.

O Ministério Público Federal - MPF - solicitou a Anatel uma verificação em 16 estações do serviço de radiodifusão em

P. E. R. Cardoso é doutorando em Engenharia Elétrica, atuando no Laboratório de Comunicações Visuais, do Departamento de Engenharia de Comunicações, da Faculdade de Engenharia Elétrica, da Universidade Estadual de Campinas (perc@decom.fee.unicamp.br).

Y. Iano é professor e coordenador do Laboratório de Comunicações Visuais, do Departamento de Engenharia de Comunicações, da Faculdade de Engenharia Elétrica, da Universidade Estadual de Campinas (yuzo@decom.fee.unicamp.br).

S. R. M. Carvalho é pesquisador no Laboratório de Comunicações Visuais, do Departamento de Engenharia de Comunicações, da Faculdade de Engenharia Elétrica, da Universidade Estadual de Campinas.

H. J. Loschi é mestrando em Engenharia Elétrica, atuando no Laboratório de Comunicações Visuais, do Departamento de Engenharia de Comunicações, da Faculdade de Engenharia Elétrica, da Universidade Estadual de Campinas eng.hermes.loschi@ieee.org).

R. F. Pinheiro e T. V. Silva são Técnico em Regulação na Coordenação de Fiscalização 3, da Gerencia Regional da Agência Nacional de Telecomunicações, em São Paulo. frequência modulada, na região metropolitana de São Paulo. Os resultados desta atividade de fiscalização podem ser obtidos no Relatório [1], onde foi verificado que 12 das 16 estações não cumprem os critérios de cobertura determinado pelo Regulamento Técnico [2]. As avaliações foram embasadas nas predições de cobertura, determinadas pela Recomendação UIT-R P.1546 [3], confrontadas com medições de intensidade de campo elétrico, de onde foi possível determinar se as coberturas reais das estações atendiam ao regulamento técnico.

Este artigo tem por objetivo apresentar as causas do não atendimento ao regulamento e das diferenças entre a intensidade de campo elétrico predita e medida, bem como alertar aos engenheiros de radiodifusão como as características de projeto afetam diretamente os parâmetros de cobertura da estação.

\section{Procedimentos de AVAliaÇão}

Para a efetiva operação das estações de radiodifusão, os regulamentos técnicos de cada serviço determinam as condições para a prestação do serviço. Primeiramente é verificado se o canal desejado pode ser incluído no Plano Básico, implicando em uma operação livre de interferências com os canais existentes. Em seguida é analisado se o canal desejado cumpre os critérios mínimos de cobertura para o município de outorga. Por fim, é verificado se as condições de projeto se enquadram no canal do Plano Básico.

Utilizando a Unidade Móvel de Radiomonitoragem, a Anatel realiza medições para verificar remotamente os parâmetros das estações de radiodifusão. Dentre os parâmetros avaliados esta a intensidade de campo elétrico, de onde é possível obter a cobertura da estação.

\section{A. Predição da Cobertura}

A Anatel utiliza as curvas de propagação da Recomendação UIT-R P.1546 [3], que é um método de predição ponto-área e que determina a distância máxima que cada intensidade de campo pode ser atingida levando-se em conta a frequência, a potência de transmissão e a altura do sistema irradiante sobre o nível médio do terreno.

A Recomendação UIT-R P.1546 [3], em seu anexo 5, apresenta diversas correções que podem ser necessárias para uma melhor predição da cobertura de uma estação. Utilizandose destas correções pode-se obter uma predição de cobertura mais próxima da realidade. A Fig. 1 apresenta a estimativa de 
cobertura de uma estação, compensada pela rugosidade do terreno, nas características máximas para o canal.

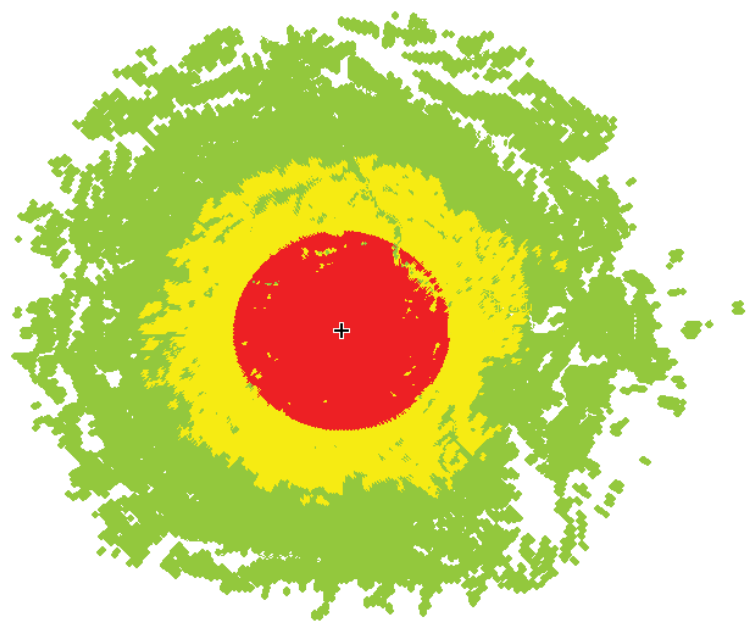

Fig. 1. Cobertura de uma estação, corrigida pelo terreno

$\mathrm{Na}$ situação apresentada na Fig. 1 temos as manchas de cobertura dos contornos de 74,66 e $54 \mathrm{~dB} \mu \mathrm{V} / \mathrm{m}$, em vermelho, amarelo e verde, respectivamente. De acordo com o Regulamento Técnico [2], a mancha em amarelo corresponde ao Contorno Protegido da estação, e indica os locais onde o serviço deve ser prestado com qualidade, isto é, livre de interferências. Observe que há granulação nestas manchas devido ao relevo e em algumas direções observa-se uma redução significativa nas distâncias. Devem-se considerar ainda os efeitos da potência efetiva irradiada e do diagrama de irradiação da antena, que com exceção da direção de máxima irradiação, atenua os sinais da estação.

A localização do sistema de transmissão é essencial, tanto que situações onde o sistema de transmissão se localiza fora do município de outorga somente são autorizadas excepcionalmente, e quando esta condição melhora a recepção no município de outorga.

Objetivando de atender com qualidade o município de outorga, os regulamentos determinam intensidades de campo mínimas para as regiões central e urbana do município. E toda emissora deve planejar seu sistema de transmissão focada neste requisito. No Serviço de FM a sede do município de outorga, que geralmente é a localização da prefeitura municipal, deve ser coberta pela intensidade de campo de $74 \mathrm{~dB} \mu \mathrm{V} / \mathrm{m}$ e a maior parte da área urbana pela intensidade de campo de $66 \mathrm{~dB} \mu \mathrm{V} / \mathrm{m}$.

A simulação de cobertura na Fig. 2 mostra um exemplo em que estas condições são atendidas. Em vermelho esta o contorno de $74 \mathrm{~dB} \mu \mathrm{V} / \mathrm{m}$ e em amarelo o de $66 \mathrm{~dB} \mu \mathrm{V} / \mathrm{m}$. Observe que a sede do município está contida no contorno de $74 \mathrm{~dB} \mu \mathrm{V} / \mathrm{m}$ e a área urbana, destacada em laranja, está contida no contorno de $66 \mathrm{~dB} \mu \mathrm{V} / \mathrm{m}$. A linha amarela não ultrapassa em nenhum ponto a linha em azul, que é o contorno de $66 \mathrm{~dB} \mu \mathrm{V} / \mathrm{m}$ teórico do Plano Básico, indicando que a estação não extrapolou os limites do canal, atendendo os requisitos. Neste caso, a propagação é calculada para cada radial, levando-se em conta a altura sobre o nível médio da radial, a potência efetivamente irradiada e o diagrama da antena.

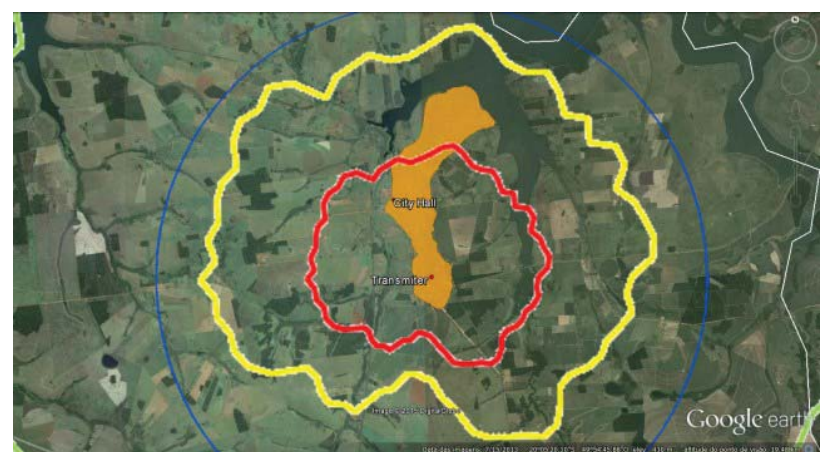

Fig. 2. Cobertura de acordo com os requisitos o regulamento técnico

A simulação apresentada na Fig. 2 é realizada com as características de projeto da estação. A diferença entre o contorno teórico, em azul, e a cobertura simulada, em amarelo, é provocada primariamente pelo relevo, e secundariamente pelas características de projeto da estação. Porém, o projeto pode ser realizado de forma a compensar as atenuações provocadas pelo relevo, e é esta discussão que será feita neste trabalho, de forma a poder contribuir com a melhora no desempenho da cobertura das estações de radiodifusão.

\section{B. Procedimento de Medição}

Para verificar os requisitos de cobertura das estações de FM, foi executado um procedimento para a medição da intensidade de campo. Para cada estação foram medidos, quando possível, três pontos na região central do município de outorga (P3, P4 e P5), sendo um na sede; em relação ao transmissor: dois pontos, mais e menos distantes, do limite urbano (P2 e P6) e; dois pontos, mais e menos distantes, do limite do município (P1 e P7). O valor obtido é a média aritmética de 100 medidas consecutivas.

A Unidade Móvel de Radiomonitoragem foi programada conforme o setup para a realização de medidas que está apresentado na Tabela I, extraída do Procedimento [4].

TABEla I Configurações de Medida

\begin{tabular}{|c|c|c|c|}
\hline \multicolumn{2}{|c|}{$\begin{array}{c}\text { JANELA DE } \\
\text { FREQUÊNCIA FIXA }\end{array}$} & \multicolumn{2}{|c|}{$\begin{array}{c}\text { JANELA DE PARÂMETROS } \\
\text { MEDIDAS UIT }\end{array}$} \\
\hline CAMPO & AJUSTE & CAMPO & AJUSTE \\
\hline Frequência & Frequência avaliada & $\begin{array}{l}\text { Arquivos de } \\
\text { salvaguarda }\end{array}$ & Nome do arquivo \\
\hline Antena & UMR - ANT184A & Tipo de Medidas & Completa \\
\hline Squelch & $6 \mathrm{~dB} \mathrm{~S} / \mathrm{R}$ & Referência $0 \mathrm{~dB}$ & Raia Máxima \\
\hline Demodulação & F3E & Modo de Medidas & Ciclos \\
\hline Ganho de RF & Normal & \multirow{2}{*}{ Ciclos } & $\mathrm{FN}=100$ \\
\hline \multirow{2}{*}{ Filtro FI } & $\mathrm{FN}=100 \mathrm{KHz}$ & & $\mathrm{M} / \mathrm{LB}=500$ \\
\hline & $\mathrm{M} / \mathrm{LB}=\mathrm{SEM}$ & FFT / Resolução & 1024 \\
\hline \multirow[b]{2}{*}{ Filtro B. Larga } & $\mathrm{FN}=\mathrm{SEM}$ & FFT / Janela & Blackman - Harris \\
\hline & $\mathrm{M} / \mathrm{LB}=300 \mathrm{kHz}$ & $\begin{array}{c}\text { Filtro Video / } \\
\text { Passa-baixa }\end{array}$ & Sem \\
\hline $\begin{array}{c}\text { Filtro } \\
\text { broadcast }\end{array}$ & Não habilitado & $\begin{array}{c}\text { Filtro Video / } \\
\text { Passa-alta }\end{array}$ & $300 \mathrm{~Hz}$ \\
\hline $\begin{array}{l}\text { Controle } \\
\text { Ganho }\end{array}$ & Médio & Limiar X dB 1 & $\mathrm{M}=15 \mathrm{~dB}$ \\
\hline \multirow[t]{2}{*}{ COR } & Inativo & Limiar X dB 2 & $\mathrm{LB}=26 \mathrm{~dB}$ \\
\hline & & Razão B & - \\
\hline
\end{tabular}

LEGENDA: M/LB= Medidas de Modulação e Largura de Banda,

FN= Medidas de Frequência e Nível,

$\mathrm{S} / \mathrm{R}=$ Relação Sinal Ruído; 


\section{RESUlTADOS}

\section{A. Localização das Estações}

Primeiramente a atividade de fiscalização localizou os pontos onde os sistemas irradiantes das estações estavam instalados. Foi confirmado que todas as 16 estações estavam fora do município de outorga. A Tabela II apresenta os municípios envolvidos com as distâncias entre a sede do município de outorga e o local de instalação do transmissor.

TABELA II MUNICÍPIOS E DISTÂNCIAS

\begin{tabular}{|l|l|c|}
\hline \multicolumn{3}{|c|}{ Distâncias de Deslocamento } \\
\hline Município de Outorga & \multicolumn{1}{|c|}{ Local de Instalação } & $\begin{array}{c}\text { Distância } \\
(\mathbf{k m})\end{array}$ \\
\hline Aruja & São Paulo & 38,9 \\
\hline Aruja & Mogi das Cruzes & 15,4 \\
\hline Atibaia & Mairiporâ & 20,3 \\
\hline Diadema & São Paulo & 14,7 \\
\hline Diadema & São Paulo & 12,7 \\
\hline Guarulhos & São Paulo & 16,6 \\
\hline Itanhaem & S. B. do Campo & 38,3 \\
\hline Itapecerica da Serra & São Paulo & 25,5 \\
\hline Itapecerica da Serra & São Paulo & 24,9 \\
\hline Itatiba & Jundiaí & 32,1 \\
\hline Mogi das Cruzes & São Paulo & 48,1 \\
\hline Osasco & São Paulo & 15,7 \\
\hline Osasco & São Paulo & 13,7 \\
\hline Santo André & São Paulo & 16,4 \\
\hline Santo André & São Paulo & 15,2 \\
\hline São José dos Campos & Mogi das Cruzes & \\
\hline
\end{tabular}

O MPF solicitou o acompanhamento de cobertura, pois nenhuma das 16 estações possui o sistema irradiante no município de outorga. Não cabe aqui discutir se estas emissoras possuem ou não autorização para instalação fora do município de outorga, somente se, nesta condição de instalação, os parâmetros de cobertura são atendidos. A Fig. 3 mostra os municípios envolvidos nesta atividade. Em verde estão os municípios onde as estações possuem outorga, em vermelho os municípios onde as estações estavam realmente instaladas, e em laranja, um caso particular, onde a estação que tinha outorga mudou-se para outro município e uma estação de outro município instalou-se.

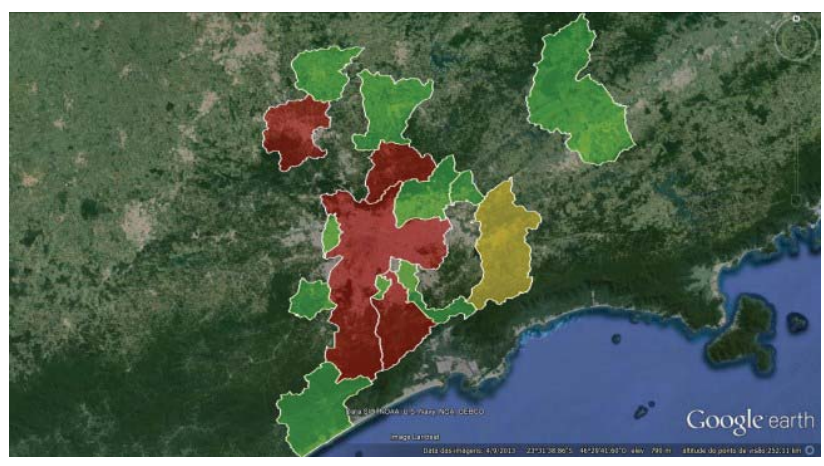

Fig. 3. Municípios envolvidos.

\section{B. Diferenças entre as Áreas de Cobertura}

A primeira análise a ser feita com a constatação da instalação fora do município de outorga é verificar o quão diferente é a área de cobertura. Neste caso foram plotadas as circunferências para o contorno protegido teórico do Plano Básico, com a estação localizada no município de outorga, em verde e no local real de instalação, em vermelho. As Fig. 4 e 5 apresentam esta análise.

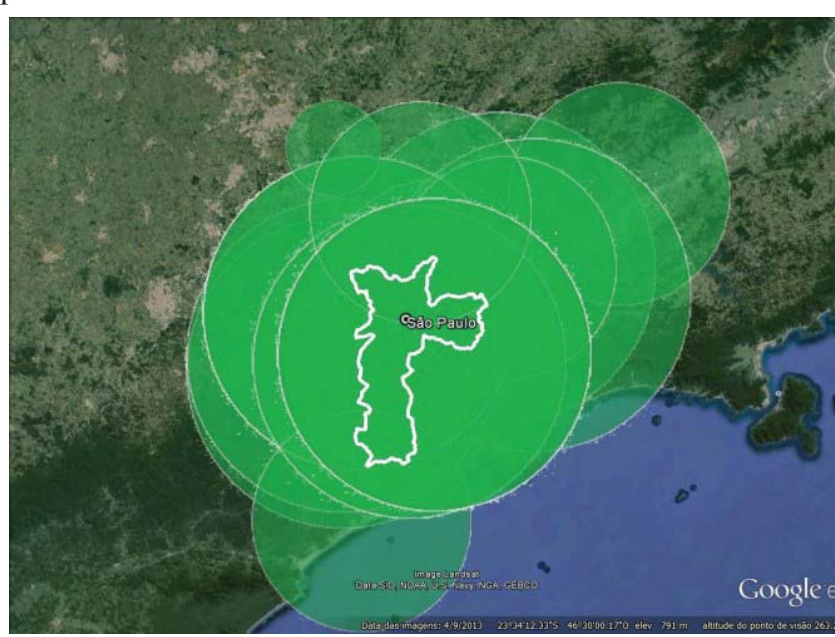

Fig. 4. Contorno Protegido Teórico, estações no município de Outorga.

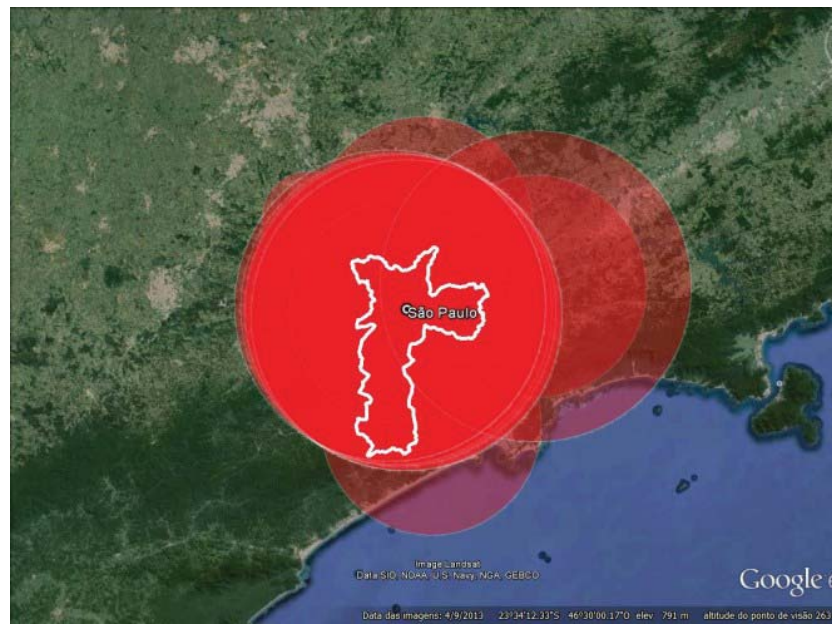

Fig. 5. Contorno Protegido Teórico, estações no local de instalação real. 
Observa-se que a região de interesse de grande parte das estações envolvidas nesta atividade de fiscalização é o município de São Paulo e os dados da Tab. II corroboram com esta constatação, sendo que 11 das 16 estações estavam instaladas no município de São Paulo.

\section{Avaliação das Coberturas}

Foram simuladas as manchas de cobertura de cada estação, tendo como parâmetro o local de instalação real e as características de Plano Básico. Foram feitas as correções em relação ao terreno, de acordo com a Recomendação UIT-R P.1546 [3]. Nestas condições é possível avaliar qual seria a cobertura máxima teórica da estação e qualquer diferença pode ser tratada como uma infração ao regulamento técnico, provocada pelas características de projeto e instalação.

Posteriormente foram confrontadas as medições em campo com as predições de cobertura. Foi elaborada uma tabela de avaliação para cada estação e com base nesta análise pode-se determinar se a estação atende ou não os requisitos do Regulamento Técnico [2]. A Tabela III exemplifica a analise para uma das estações.

TABELA III AVALIAÇÃO DA COBERTURA

\begin{tabular}{|c|c|c|c|}
\hline \multicolumn{4}{|c|}{ Exemplo de avaliação de uma Estação } \\
\hline Ponto & Previsto $(\mathrm{dB} \mu \mathrm{V} / \mathrm{m})$ & Medido $(\mathrm{dB} \mu \mathrm{V} / \mathrm{m})$ & Situação \\
\hline P1 & $\mathrm{NC}$ & 44,72 & NA \\
\hline $\mathrm{P} 2$ & 66 & 61.33 & a \\
\hline P3 & 74 & 72.67 & 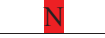 \\
\hline P4 & 66 & 49.23 & $\mathrm{~N}$ \\
\hline P5 & 74 & 78.96 & A \\
\hline P6 & $\mathrm{NC}$ & --- & --- \\
\hline P7 & $\mathrm{NC}$ & 50.93 & NA \\
\hline
\end{tabular}

Dos sete pontos avaliados, apenas quatro (P2, P3, P4 e P5) tem intensidade de campo mínima determinadas pelo regulamento, sendo que três $(\mathrm{P} 2, \mathrm{P} 3$ e $\mathrm{P} 4)$ não atenderam os requisitos de cobertura. Assim, entendeu-se que a estação não cumpre o Regulamento Técnico. O mesmo ocorreu com mais 11 estações das 16 avaliadas [1].

A Anatel tem a função de fiscalizar e autuar as entidades, cabendo à equipe técnica da entidade fiscalizada, no caso de uma irregularidade, buscar as causas e soluções pertinentes para a eliminação da irregularidade.

\section{Diferenças entre a Predição e a Medição}

Cabe discutir a respeito das diferenças entre as predições de cobertura e as medições. De modo geral, apenas 29 dos 88 pontos medidos apresentaram uma intensidade de campo superior ao previsto. A Fig. 6 mostra as avaliações para os 88 pontos, que abrangeram as 16 estações.

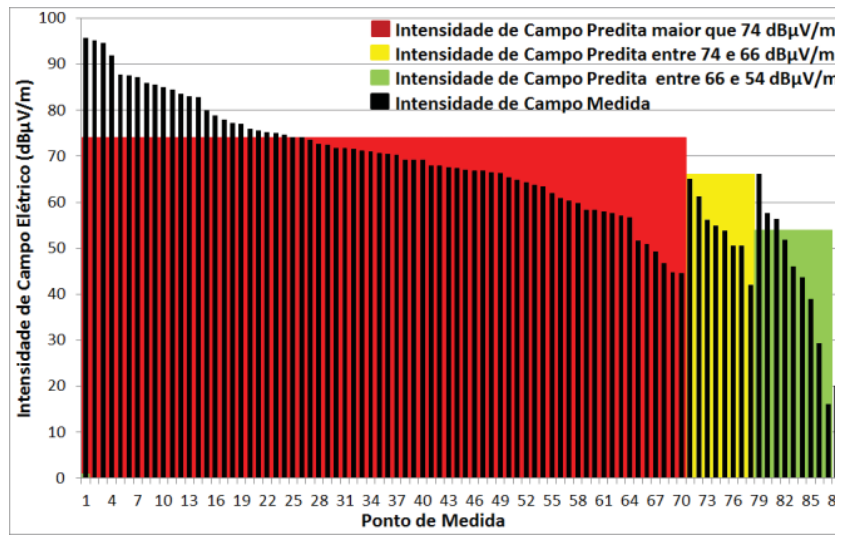

Fig. 6. Diferenças entre a Predição e a Medição.

A maioria das emissoras envolvidas é de classe especial, isto é, tem potencia de operação superior aos $50 \mathrm{~kW}$ e raio de cobertura superior aos $40 \mathrm{~km}$. Desta forma, apesar do sistema irradiante estar fora do município de outorga, a quase totalidade dos municípios estaria coberta pelo campo de $74 \mathrm{~dB} \mu \mathrm{V} / \mathrm{m}$. Na Fig. 6 observa-se que dos 88 pontos, 70 estão com previsão de campo elétrico superior a este valor, fundo em vermelho.

Analisando as diferenças entre as coberturas previstas e medidas, principalmente na maioria dos pontos que está abaixo da intensidade de campo prevista, podemos analisar quais são as causas deste não atendimento. A seguir são apresentadas algumas simulações que podem demonstrar que as características de projeto e de operação contribuem de forma significativa para este fenômeno.

\section{SimUlaÇÕES DE PROJETO DE UMA ESTAÇÃO}

Diversos fatores podem modificar a cobertura de uma estação de radiodifusão além do relevo. Como citado anteriormente, o local de instalação, o diagrama da antena, a potencia irradiada e a altura do sistema irradiante são fundamentais para uma boa cobertura.

Para demostrar isto, foi utilizado o SIGAnatel [5] e [6] para simular uma estação de FM, no canal 250 (97,9 MHz), classe A4, no município de Campinas/SP. A potência de transmissor de 1,0 kW; altura do centro de fase da antena de $65 \mathrm{~m}$; ganho da antena de $3 \mathrm{~dB}$, comprimento da linha de transmissão de $70 \mathrm{~m}$, com atenuação de $0,4 \mathrm{~dB} / 100 \mathrm{~m}$; e demais perdas de $1 \mathrm{~dB}$.

Em Campinas/SP há dois locais onde se concentram as estações de radiodifusão, o bairro São Gabriel (22 ${ }^{\circ} \mathrm{S} 56^{\prime} 37^{\prime \prime}$ / $47^{\circ} \mathrm{W} 01^{\prime}$ 50") e o bairro Castelo (22 ${ }^{\circ} \mathrm{S} 53^{\prime} 24^{\prime \prime} / 47^{\circ} \mathrm{W} 04^{\prime}$ 37 '). Inicialmente foi simulada a cobertura com o diagrama omnidirecional teórico nestes dois locais para que somente os efeitos do terreno fossem observados e a partir deste resultado, escolhido o local para as demais simulações.

TABELA IV COBERTURA PELA COORDENADA GEOGRÁFICA

\begin{tabular}{|c|c|c|}
\hline \multicolumn{3}{|c|}{ Comparação de Cobertura } \\
\hline Local & São Gabriel $\left(\mathbf{k m}^{2}\right)$ & Castelo $\left(\mathrm{km}^{2}\right)$ \\
\hline Area de Cobertura & $1.100,43$ & $1.074,21$ \\
\hline
\end{tabular}


Com as características de projeto das duas estações idênticas, apenas alterando-se a coordenada geográfica dentro do município de Campinas, obteve-se o melhor resultado, apesar da pequena diferença, no São Gabriel, Tabela IV. Nestas condições foi possível obter uma cobertura que atende aos requisitos de enquadramento do Regulamento Técnico [2], isto é, não há extrapolação em nenhuma radial. A Fig. 7 apresenta o resultado desta simulação.

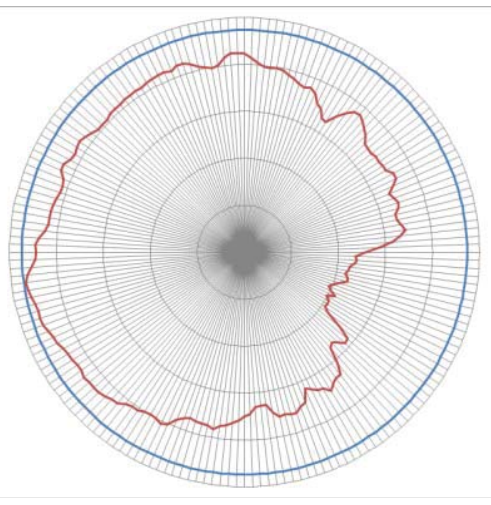

Fig. 7. Simulação de Cobertura - Referência do Terreno.

Pode-se observar a grande perda da cobertura provocada pelas irregularidades do terreno. A área de cobertura resultante, em vermelho, é $62,50 \%$ da área da circunferência teórica, em azul, de uma emissora classe A4.

Partindo-se para casos reais, foram simuladas as coberturas para duas antenas comerciais [7] e [8] com diagramas de irradiação diferentes, apontadas para o norte verdadeiro $\left(0^{\circ} \mathrm{NV}\right)$. Tomando-se como referencia a simulação com a antena omnidirecional teórica, obtiveram-se os seguintes resultados, apresentados na Tabela V.

\section{TABELA V COBERTURA PELO Diagrama DA ANTENA}

\begin{tabular}{|c|c|c|}
\hline \multicolumn{3}{|c|}{ Comparação de Cobertura } \\
\hline Diagrama & Referência & Teórico \\
\hline Omnidirecional & $100 \%$ & $62,50 \%$ \\
\hline Antena 1 & $95,76 \%$ & $59,85 \%$ \\
\hline Antena 2 & $88,98 \%$ & $55,61 \%$ \\
\hline
\end{tabular}

Com as simulações destas duas antenas, comparando-se com a cobertura de referência da antena omnidirecional verificamse perdas de 4,24 e $11,02 \%$ da cobertura. Em relação à cobertura ideal do canal, as perdas foram de 2,65 e $6,89 \%$. Estas perdas podem ser reduzidas alterando-se o apontamento da antena.

Foram simulados os apontamentos onde a máxima irradiação do diagrama era direcionada para a radial de menor propagação $\left(110^{\circ}\right)$, que no caso seriam os azimutes $185^{\circ} \mathrm{NV}$ e $25^{\circ} \mathrm{NV}$ para [7] e [8], respectivamente. Do mesmo modo, simularam-se os apontamentos onde a máxima irradiação do diagrama era direcionada para a radial de maior propagação $\left(260^{\circ}\right)$, que no caso seriam os azimutes $335^{\circ} \mathrm{NV}$ e $175^{\circ} \mathrm{NV}$ para [7] e [8], respectivamente. Os resultados destas simulações estão na Tabela VI.
TABELA VI COBERTURA PElo AZIMUte

\begin{tabular}{|c|c|c|}
\hline \multicolumn{3}{|c|}{ Comparação de Cobertura } \\
\hline Diagrama & Max $\mathbf{1 1 0}^{\boldsymbol{}}$ & Max $\mathbf{2 6 0}^{\circ}$ \\
\hline Antena 1 & $59,35 \%$ & $59,90 \%$ \\
\hline Antena 2 & $55,21 \%$ & $55,87 \%$ \\
\hline
\end{tabular}

Mudando-se o apontamento da antena é possível alterar o percentual de cobertura. Observa-se que o apontamento onde a máxima irradiação do diagrama era direcionada para a radial de maior propagação $\left(260^{\circ}\right)$ apresentou melhores resultados para os dois fabricantes.

Analisando uma situação de queda de rendimento do transmissor, em que a potência efetiva irradiada sofra uma redução de 90 e de $50 \%$, podemos simular qual será a que da percentual da cobertura. Para estas simulações, vamos comparar com o diagrama de irradiação [7], apontado para o azimute $335^{\circ} \mathrm{NV}$, que corresponde a $59,90 \%$ da cobertura ideal do canal.

TABELA VII COBERTURA PELA POTÊNCIA DO TRANSMISSOR

\begin{tabular}{|c|c|c|}
\hline \multicolumn{3}{|c|}{ Comparação de Cobertura } \\
\hline Diagrama & $\mathbf{0 . 9} \boldsymbol{T X}$ & $\mathbf{0 . 5} \boldsymbol{T X}$ \\
\hline Antena 1 & $56,98 \%$ & $42,56 \%$ \\
\hline
\end{tabular}

O resultado da simulação com o diagrama de irradiação da Antena 1, apontado para o azimute $335^{\circ} \mathrm{NV}$ ainda pode ser melhorado, aumentando-se a altura da antena. Porém simplesmente aumentar a altura provoca uma extrapolação dos limites teóricos para a classe A4. Para contornar esta extrapolação pode-se recorrer a limitações no diagrama do sistema irradiante. Simulando a estação com altura de antena de $150 \mathrm{~m}$ e com limitação da potência efetiva irradiada de $50 \%$ entre os azimutes $205^{\circ}$ a $320^{\circ}$ e de $80 \%$ entre os azimutes de $320^{\circ}$ a $360^{\circ}$ e de $0^{\circ}$ a $5^{\circ}$, obtemos uma cobertura correspondente a $78,74 \%$ da cobertura ideal da classe A4. A Fig. 8 apresenta o resultado desta simulação.

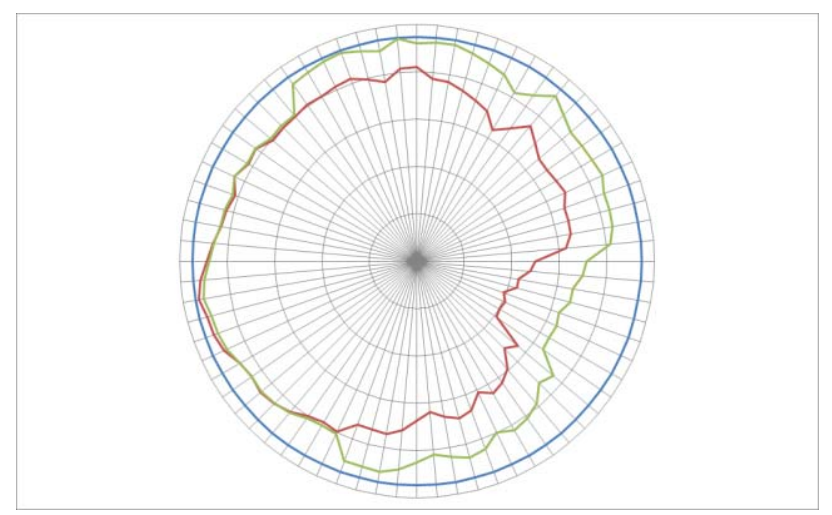

Fig. 8. Simulação com Melhora na Cobertura.

A Fig. 8 apresenta, em vermelho, a cobertura prevista para a simulação com o diagrama de irradiação [7], apontado para o azimute $335^{\circ} \mathrm{NV}$, a $65 \mathrm{~m}$ de altura, que corresponde a uma cobertura de $59,90 \%$ da cobertura ideal do canal, em azul. Aumentando a altura do sistema irradiante e limitando o 
diagrama conforme indicado anteriormente, temos a cobertura representada em verde. Observa-se uma significativa melhora de $16,24 \%$ na cobertura da emissora, apenas trabalhando com o sistema irradiante mais próximo ao ideal, com limitações; apontado para a direção de melhor propagação; e altura da antena adequada.

\section{AGRADECIMENTOS}

Os autores gostariam de agradecer à CAPES, ao CNPq, e à Fapesp. Também aos demais professores e funcionários do DECOM, da FEEC e da Unicamp.

\section{CONCLUSÕES}

A partir das avaliações das coberturas de 16 estações de FM na Região Metropolitana de São Paulo, onde se verificou que 12 destas não cumprem com os requisitos de cobertura, podese analisar as diferenças entre as predições e as medições em campo.

Apesar de ser um tema estudado há bastante tempo, os resultados da avaliação das emissoras demonstram que ainda existem falhas nos projetos das estações, que prejudicam a prestação do serviço com qualidade.

Tais diferenças, que tem grandes contribuições do relevo, mas que também podem ser provocadas pelas características de projeto e operação, que são de responsabilidade dos engenheiros das estações.

Foi demostrado, por meio de simulações, que um pouco de trabalho sobre as características do projeto pode melhorar o desempenho da cobertura, inclusive compensando as atenuações do relevo.

\section{REFERÊNCIAS}

[1] "Relatório de Fiscalização no 0277/2013/ER01FT", Agência Nacional de Telecomunicações, São Paulo, Brasil, March 2013.

[2] "Resolução Anatel no 67-Regulamento Técnico para Emissoras de Radiodifusão Sonora em Freqüência Modulada", Agência Nacional de Telecomunicações, Brasília, Brasil, November 1998.

[3] Recommendation ITU-R P.1546 - Method for point-to-area predictions for terrestrial services in the frequency range $30 \mathrm{MHz}$ to $3000 \mathrm{MHz}$, October, 2009.

[4] Procedimento de Fiscalização de Gestão e Monitoramento do Espectro de Radiofrequência - FIS.PF.041, January, 2010.

[5] SIGANATEL - Sistema de Informações Geográficas (Ferramenta para Calculo de Viabilidade de Canais de Radiodifusão de TV e FM). Disponível em: http://sistemas.anatel.gov.br/siganatel/.

[6] P. H. F. Silva, M. G. Passos, "Analise Numérica da Ferramenta SIGANATEL para o Calculo de Viabilidade de Canais de FM", XXV SIMPOSIO BRASILEIRO DE TELECOMUNICAÇÕES - SBrT 2007, 03-06 September 2007, RECIFE, PE.

[7] Antenna FM 01 http://www.idealantenas.com.br/produtosport/fm/PDF\%20Antena $\% 20 \mathrm{~F}$ MV.pdf .

[8] Antenna FM 02 http://teeltele.com/equipamentos/antenas-de-fmpolarizacao-circular/.

[9] E. S. Bueno, G. Bedicks Jr., C. Akamine, E. L. Horta, "Results of Field Tests of the ISDB-TB System at $8 \mathrm{MHz}$ in Botswana" Revista de Radiodifusão, v.07 n.08 2013, São Paulo, SP.

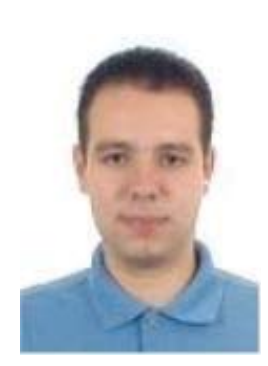

Paulo E. R. Cardoso (M'15) Possui graduação em Engenharia Elétrica pela FEEC-Unicamp (2002) e mestrado em Engenharia Elétrica (Eletrônica) pelo DEMIC-FEEC-Unicamp (2005).

Atualmente é Doutorando no DECOMFEEC-Unicamp, pesquisando TV Digital. Está licenciado do cargo de Especialista em Regulação da Agência Nacional de Telecomunicações - Anatel, onde atua na Coordenação de Outorga e Recursos à Prestação, na Gerência Regional da Anatel em São Paulo, trabalhando com o Licenciamento e Alteração de Características Técnicas das estações de Radiodifusão. Anteriormente, atuou na Fiscalização Técnica em entidades de Radiodifusão e na solução de problemas de radiointerferência em qualquer sistema de telecomunicação. Foi responsável pelo Regulamento Técnico para Emissoras de Radiodifusão Sonora em Frequência Modulada e pela análise de processos de viabilidade técnica para inclusão ou alteração do Plano Básico de Distribuição de Canais de Radiodifusão Sonora em Frequência Modulada. Participou como observador do Governo Federal nos testes de Radiodifusão Sonora Digital, tanto nos testes do padrão americano - HD Radio, em 2008 e 2012, como nos testes do padrão europeu - DRM, em 2010. Atuou como Pesquisador de Telecomunicações da Fundação Centro de Pesquisa e Desenvolvimento em Telecomunicações - CPqD.

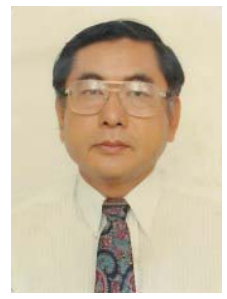

Yuzo Iano Possui graduação em Engenharia Elétrica pela Unicamp (Universidade Estadual de Campinas 1972), mestrado em Engenharia Elétrica pela Unicamp (1974) e doutorado em Engenharia Elétrica pela Unicamp (1986). Atualmente é Professor Titular MS6 do Decom/Feec/Unicamp (Departamento de Comunicações da Faculdade de Engenharia Elétrica e de Computação da Unicamp).

Tem experiência na área de Engenharia Elétrica, com ênfase em Telecomunicações, Eletrônica e Tecnologia da Informação, atuando principalmente no campo das comunicações audiovisuais e de dados. Os principais temas de interesse são: processamento e transmissão digital de sinais/imagens/vídeo/áudio/dados, hdtv, tv digital, redes 3G/4G/5G, middleware, transmissão/canalização/radiodifusão de sinais de televisão, reconhecimento de padrões, codificação digital de sinais, transmissão/armazenamento de dados e cidades inteligentes/digitais.

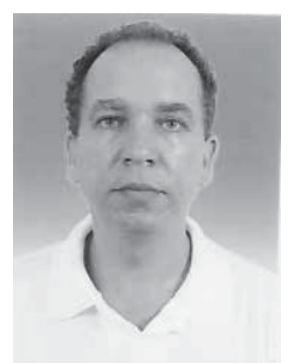

Silvio R. M. Carvalho Possui graduação em Engenharia Elétrica pela FEECUnicamp, Mestre em Telecomunicações e Telemática pela FEEC-DECOMUnicamp, Doutor em Telecomunicações e Telemática pela FEEC-DECOMUnicamp, pesquisador do LCV-Unicamp especialista nas áreas de TV Digital, Redes SFN.

Engenheiro de Projetos de Infraestrutura para Televisão na EPTV. 


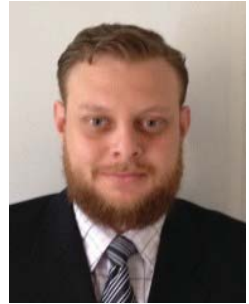

Hermes J. Loschi Formado em Engenharia de Controle e Automação (Universidade Paulista - 2014). Atualmente, M.Sc Candidato pelo Decom / Feec / Unicamp (Departamento de Comunicações da Faculdade de Engenharia Elétrica e de Computação da Unicamp).

Atualmente, um pesquisador do laboratório de comunicações visuais, departamento de comunicação. Os principais temas de interesse são: Wireless Network, TV digital, células e sistemas fotovoltaicos, energia e rastreamento solar.

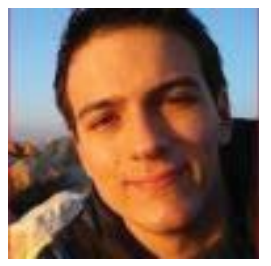

Rafael F. Pinheiro Possui especialização em Direito Público sobre o tema "Espectro de radiofrequências como bem público" pela Pontifícia Universidade Católica de Minas Gerais (2014), graduação em Relações Internacionais pela Universidade de São Paulo (2009) e formação de nível técnico em Eletrônica pelo Centro Federal de Educação Tecnológica de São Paulo (2004).

Desde 2005, exerce cargo de Técnico em Regulação na Agência Nacional de Telecomunicações - Anatel, trabalhando na área de fiscalização técnica e resolução de radiointerferências da Gerência Regional em São Paulo. Anteriormente, atuou na Gerência Operacional de Outorga e no Centro Regional do Sistema de Gestão e Monitoragem do Espectro (SGME) em São Paulo. Participou como observador nos teses de Radiodifusão Sonora Digital com o padrão europeu (DRM), em 2010. Em 2012, participou do programa "Spectrum Management Sequence", organizado pela USTTI, FCC e NTIA, nos Estados Unidos da América, sobre a regulação, gestão e monitoragem do espectro de radiofrequências naquele país.

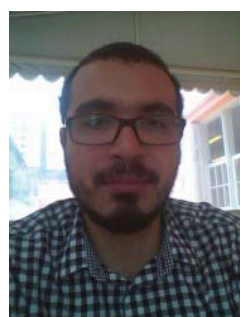

Thiago V. Silva Possui especialização em Automação Industrial pela Faculdade SENAI de Tecnologia Mecatrônica, com temas correlatos a instrumentação e controle ótimo (2008), graduação em Mecatrônica Industrial pela Faculdade de Tecnologia Termomecânica (2005) e atualmente estuda Bacharelado em Ciência e Tecnologia pela Universidade Federal do ABC. Atua, desde 2009, como Técnico em Regulação na Agência Nacional de Telecomunicações, Anatel, desempenhando atividades relacionadas a controle e uso do espectro, fiscalização técnica de entidades e resolução de conflitos em radiointerferência.
Cite this article:

E. R. Cardoso, P., Iano, Y., R. M. Carvalho, S., J. Loschi,H., F. Pinheiro, Rafael e V. Silva, T.; 2015. Técnicas de Projeto para Atendimento aos Requisitos de Cobertura de Estações de FM. SET EXPO PROCEEDINGS. ISSN Print: 2447-0481.ISSN Online: 2447-049X. v.1. doi: 10.18580/ setep.2015.1.6 Web-link: http://dx.doi.org/10.18580/setep.2015.1.6 